\title{
Severe adenovirus pneumonia in immunocompetent adults: a case report and review of the literature
}

\author{
F. A. Hakim • I. M. Tleyjeh
}

Published online: 21 November 2007

(C) Springer-Verlag 2007

\begin{abstract}
Adenovirus is a frequent cause of mild self-limiting upper respiratory tract infection, gastroenteritis, and conjunctivitis in infants and young children. Fatal infections (severe pneumonia progressing to respiratory failure, septic shock and/or encephalitis) are rare among immunocompetent adults. We report a case of severe adenovirus pneumonia in a young immunocompetent male who presented with sudden onset respiratory distress that progressed rapidly to respiratory failure and made a successful recovery on supportive measures. Systematic review of the literature identified 14 cases of severe adenovirus pneumonia (defined as respiratory failure requiring ventilatory support at any point during the course of illness) in otherwise healthy immunocompetent adults both in epidemic and community settings. We describe the clinical characteristics, radiological features, and outcome of identified cases.
\end{abstract}

\section{Introduction}

Adenovirus is a frequent cause of mild self-limiting upper respiratory tract infection, gastroenteritis, and conjunctivitis

F. A. Hakim $(\bowtie)$

Department of Internal Medicine, 4th Floor, Main Hospital,

King Fahd Medical City,

P.O. Box 59046, Riyadh 11525, Saudi Arabia

e-mail: fayazhakim@hotmail.com

I. M. Tleyjeh

Division of Infectious Diseases, King Fahd Medical City,

Riyadh, Saudi Arabia

I. M. Tleyjeh

Division of Infectious Diseases, Mayo Clinic,

College of Medicine,

Rochester, MN, USA in infants and young children. Epidemics of self-limited infections by adenovirus (especially serotype 4 ) have been reported from time to time in military recruits. Severe adenovirus infections causing significant morbidity and mortality are well described in immunocompromised patients but are extremely rare in healthy immunocompetent adults [1]. Among the known 51 serotypes of human adenoviruses, serotypes 3 and 7 account for most of these severe infections. Such infections first gained attention during the epidemics of the 1960s and early 1970s in military trainees, when no vaccine was available $[2,3]$. Occurrence of such infections among the military recruits dropped significantly with the introduction of a vaccine in 1971. However, severe adenovirus infections among immunocompetent adults raised concern when Ryan et al. reported related deaths in two military recruits in 2001 [4]. Sporadic cases of severe infection have been reported from the community as well $[5,6]$.

We report a case of severe adenovirus pneumonia in a previously healthy immunocompetent male who presented to us with rapidly developing respiratory failure and made a successful recovery on supportive measures. We also summarize the clinical features, radiological findings, and outcome of severe adenovirus pneumonia cases that have been reported in the literature.

\section{Case report}

A 16-year-old Saudi male student reported to our emergency room (ER) with a 1-day history of sudden onset dry cough and shortness of breath. He denied fever, chest pain, hemoptysis, palpitation, or leg swelling. Review of systems was unremarkable. He was a smoker and denied use of alcohol and drugs. He had no risk factors for human 
immunodeficiency virus (HIV). There was no history of travel outside Riyadh city in the recent past. He had no exposure to birds or contact with sick persons. He had no pets at home. Past medical history and family were unremarkable.

On his arrival to the ER, the patient was febrile (temperature $38.3^{\circ} \mathrm{C}$ ), tachypneic (respiratory rate $24 / \mathrm{min}$ ), and tachycardic (pulse rate 120/min). His blood pressure was $130 / 78 \mathrm{mmHg}$ and oxygen saturation was $78 \%$ by pulse oximetry while breathing room air. There was mild pharyngeal and tonsillar erythema but no conjunctival injection, rash, or lymphadenopathy. There was no lower limb edema or jugular venous distension. Cardiovascular examination was normal and auscultation of the chest revealed bilateral scattered rhonchi. The rest of the systemic examination was unremarkable.

Laboratory investigations at admission revealed polymorpholeukocytosis (white cell count $28.3 \times 10^{9} / \mu$ l with 94.7\% neutrophils). Hemoglobin $(15.5 \mathrm{~g} / \mathrm{dl})$, platelets $\left(230 \times 10^{9} / \mu \mathrm{l}\right)$, sedimentation rate $(15 \mathrm{~mm} 1 \mathrm{st} h)$, and urinalysis were normal. Chest X-ray was normal and arterial blood gases revealed hypoxemia and mild respiratory alkalosis. Serum biochemical panel including random glucose, urea/electrolytes, liver function tests, and amylase were normal. Serum cardiac enzymes and coagulation profile were normal. Doppler ultrasound of leg veins was negative for venous thrombosis and ventilation perfusion scan revealed multiple bilateral ventilation perfusion defects. Computed tomogram (CT) scan of the chest showed no parenchymal lung disease and spiral CT angiogram scan did not reveal pulmonary embolism.

Six hours after presentation, the patient developed worsening respiratory distress and was transferred immediately to the intensive care unit (ICU) and mechanically ventilated. Given the septic shock picture, empiric antimicrobial therapy was started. Over the next $72 \mathrm{~h}$ the patient's respiratory failure worsened and he developed fluidresistant shock. Inotropes and vasopressors were used to maintain his mean arterial pressure between 60 and 70 $\mathrm{mmHg}$. He continued to have low-grade fever, developed new conjunctival congestion, and had coarse crackles on chest auscultation. Repeat portable chest X-ray showed bilateral fluffy and confluent air space shadowing in the mid and lower zones. CT scan of the chest was consistent with bilateral consolidation and bilateral pleural effusion. Transesophageal echocardiography was normal. Cultures drawn at admission were negative. Legionella urine antigen, Mycoplasma serology, and HIV serology were negative. Gram staining, acid fast staining, Mycobacterium tuberculosis DNA probe, cytomegalovirus, and Pneumocystis carinii performed on bronchoalveolar lavage were negative. Repeat cultures and urine drug screen were negative. The patient developed nonoliguric acute renal failure. Urinalysis was negative for active urine sediment and proteins and renal ultrasound was normal. Immunologic tests including ANA, anti-dsDNA, c-ANCA, and pANCA and cryoglobulins were negative. Interestingly, the patient developed bilateral conjunctival chemosis and hemorrhages with mild watery discharge on day 6 of illness (Fig. 1). Tracheal and nasopharyngeal aspirates sent for immunofluorescent viral studies were reported as strongly positive for adenovirus (Magen direct antibody fluorescence). Adenovirus serology and serotyping were not performed. Droplet precautions were observed to prevent infection of the caring staff. Ophthalmological evaluation revealed hemorrhagic conjunctivitis. The systemic empirical antibiotics were withdrawn and supportive measures for respiratory and renal failure continued with continuous monitoring of vitals and serum biochemical panels. No antiviral therapy was used. Inotropes and vasopressors were gradually withdrawn. The patient's respiratory failure and renal failure improved over the next 7 days and he was successfully weaned off the ventilator and extubated. He was discharged home on day 18 after admission. He was doing very well at the 6-month follow-up.

\section{Materials and methods}

We reviewed and analyzed the clinical features, radiological findings, and outcome of severe adenovirus pneumonia in immunocompetent patients reported between 1971 and 2006 by searching MEDLINE for English reports and using "severe," "adenovirus," "pneumonia," and "immunocompetent" as search terms. We defined severe adenovirus pneumonia if associated with respiratory failure requiring ventilatory support at any point during the course of illness and immunocompetent adults as individuals with no acquired or congenital immunodeficiency state with or without associated premorbid conditions. We identified 14 cases. Seven of these cases occurred in epidemics among

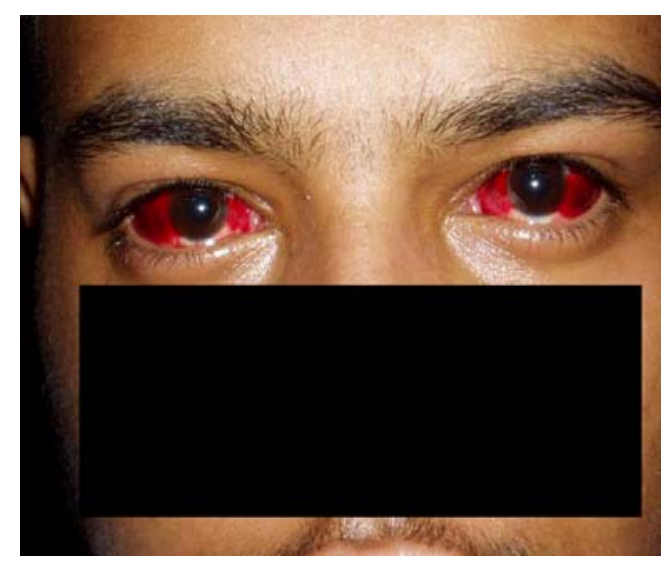

Fig. 1 Photograph of the patient showing bilateral hemorrhagic conjunctivitis 
military recruits and a mental health care center and the remaining seven were reported from the community.

\section{Results}

Table 1 summarizes age, sex, presenting complaints, physical findings, radiological findings, and outcome of each patient. Adenovirus serotype (wherever performed), time to ventilation, and time to death from onset of illness are also included when available. The majority of the patients $(8 / 12,66.66 \%)$ were males and the mean age was 32.75 years (range: $19-56$ years). Most patients had no premorbid conditions. Respiratory symptoms were present in all patients; three had associated diarrhea. Radiograph of the chest revealed focal or diffuse infiltrate or consolidation. Serotyping was done in six patients: three had serotype 7 , three had serotype 3 , and one had both serotypes 4 and 7 . Mean time from onset of symptoms to admission to ICU for respiratory failure was 7.5 days (range: 2-17 days). Of the 14 patients identified, $8(57.14 \%)$ died due to worsening respiratory failure. Three of the deceased patients had associated renal failure and disseminated intravascular coagulopathy (DIC) and one had meningitis. An antiviral agent (cidofovir) was used in one patient. Most of these patients developed hypotension during the course of their disease and six of these progressed to fluid-resistant shock.

\section{Discussion}

Adenovirus is a frequent cause of mild upper respiratory tract illness. Severe adenovirus infection is rare in immunocompetent adults. Outbreaks of fulminant respiratory illness due to adenovirus have been reported in military camps in 1962 and 1972 [2, 3], in hospitalized patients in 1978 [7], in a mental health care center in 1996 [8], and among civilians in 1998 [9]. Several sporadic cases of adenovirus infections in healthy immunocompetent individuals with case fatalities have also been reported $[5,6]$.

Our review suggests that most patients have upper respiratory symptoms prior to the onset of respiratory distress and progression to respiratory failure is unpredictable, occurring over hours to days. The presence of flu-like symptoms, diarrhea, and conjunctival infection may be a clue to the viral etiology of the illness. Hemorrhagic conjunctivitis was present in only one of the identified patients; when present it is strongly associated with adenovirus infection. However, it may not be apparent early in the course of the disease as is illustrated in our case. The majority of such patients are febrile and tachycardic at presentation. Chest auscultation may be normal or may reveal localized or bilateral rales, rhonchi, or signs of consolidation. Fluid responsive hypotension and a transient drop in leukocyte count (relative leukopenia and lymphopenia) are common during the course of illness. Initial chest X-ray may be normal but reveal focal reticulonodular infiltrate or a lobar consolidation with the progression of the infection. Lobar consolidation is rare in other types of viral pneumonia [5-8]. The majority of patients with severe adenovirus infection died due to respiratory failure and shock. Disseminated intravascular coagulopathy, renal failure, and fluid-resistant shock are also common causes of death and should be always looked for in such patients. Meningitis is a rare but fatal complication of severe adenoviral pneumonia. The whole clinical scenario may be mistaken for bacterial sepsis. Whenever considered, the diagnosis of adenovirus infection is established by isolating the virus on cultures or detecting viral particles on fluorescence antibody testing from material obtained from nasopharyngeal and tracheal aspirate, bronchoalveolar lavage, or lung biopsy. A fourfold or more rise in adenovirus-specific antibody titers during the course of illness suggest acute infection and alternatively establishes the diagnosis. Treatment of severe adenovirus infection is supportive and no antiviral agent has been shown to alter the unpredictable course of the disease. Cidofovir and intravenous immunoglobulin have been used with some success in patients with liver and bone marrow transplant [10]. In our review, it was used in only one patient who did not survive. It appears that severe adenovirus infections even in immunocompetent patients are associated with high mortality and morbidity. The spectrum of presentation and characteristics of severe adenovirus infections could be affected by publication bias.

Adenovirus produces cytolysis which accounts for tissue damage and subsequent dysfunction of the affected organs. It also induces interleukin 8 (IL-8) production, which causes a severe systemic inflammatory response causing systemic vasodilatation and triggers DIC. Damage to pulmonary capillaries also contributes to respiratory failure. Cellular immunity is important in viral infection in general. Hence, severe adenovirus infections are common in patients with defective or deficient T-cell immunity such as those with allogenic stem cell transplantation, solid organ transplants, and HIV infection. Infants and young children also are at risk of severe adenovirus infections due to immature T-cell function. Why severe adenovirus infection should occur in immunocompetent individuals is not well understood at this point in time. A unique interplay between viral factors (virulence) and host factors (genes) have been well described in adenovirus infections. Virulent strains of adenovirus (serotypes 3 and 7 , etc.) in a genetically susceptible host impair cytokine, T-cell function, and expression of major histocompatibility complex expression 


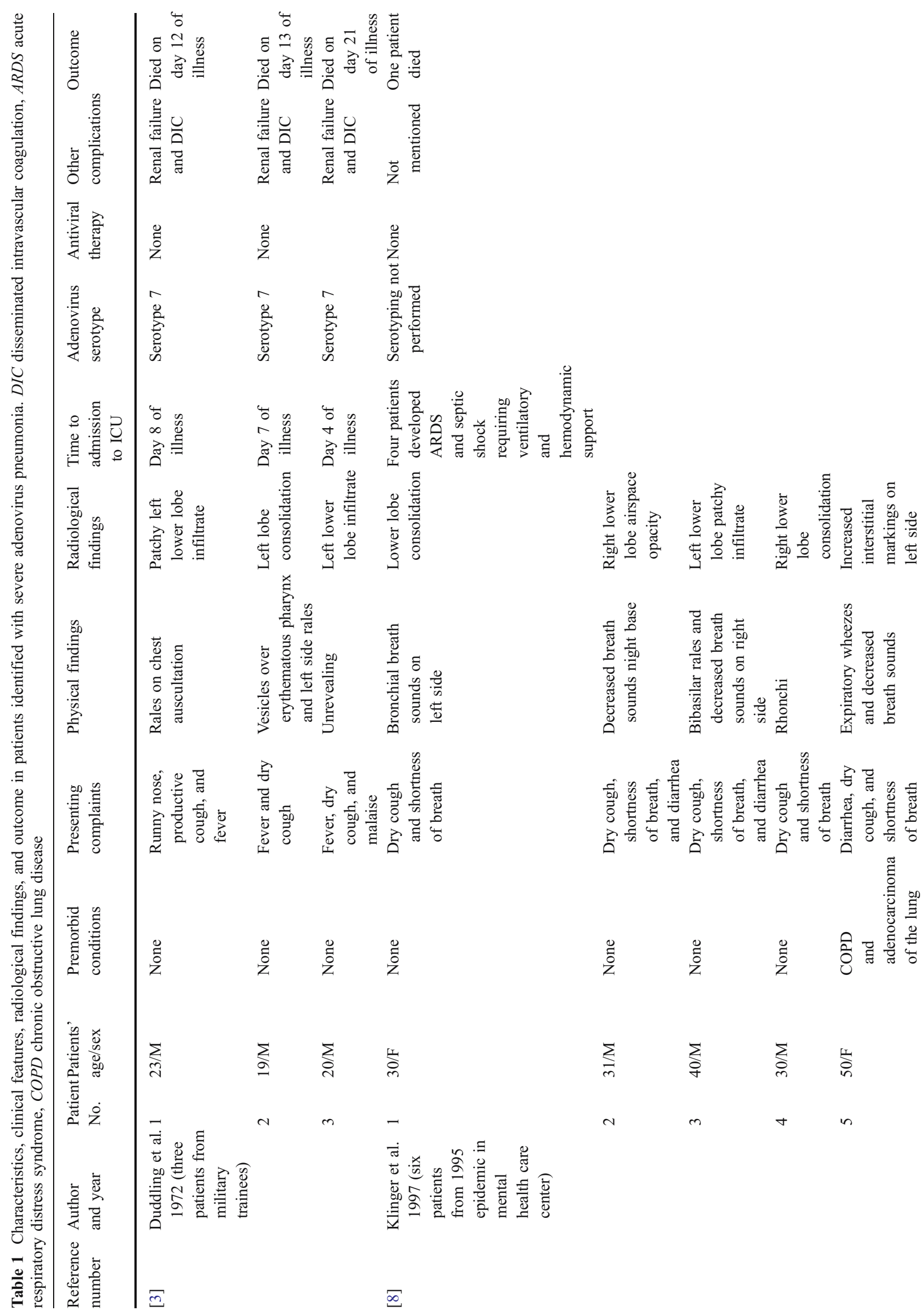




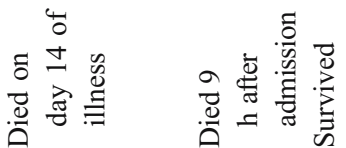

帚

芩

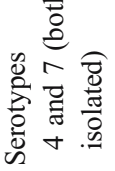

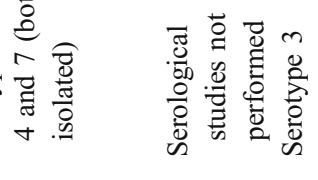

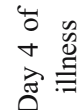

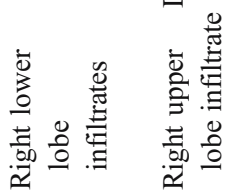

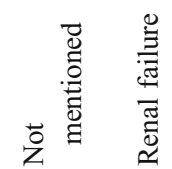

خे

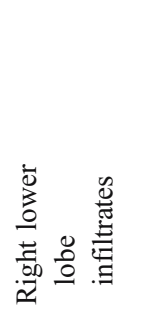

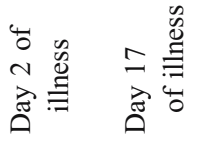

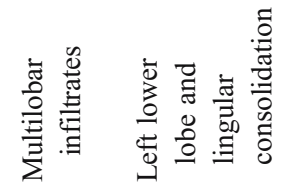

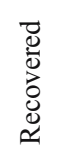

艺莺离离

焉

言

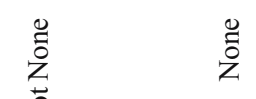

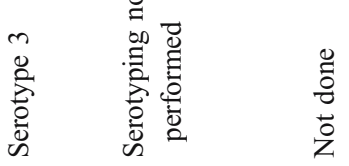

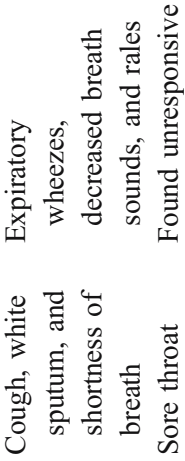

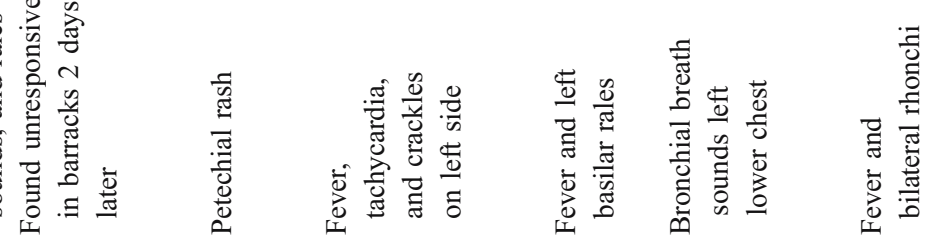

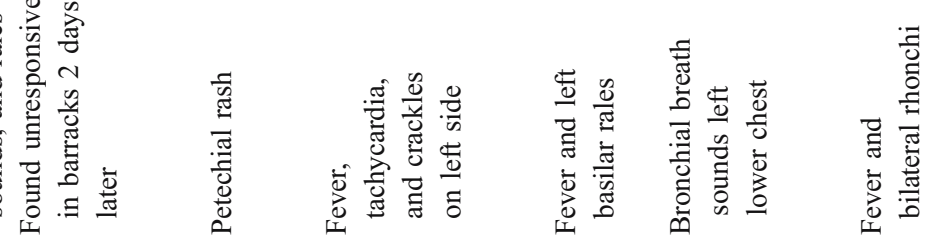

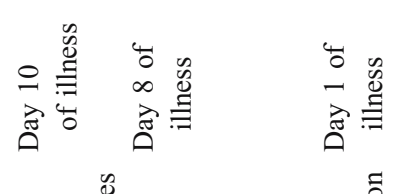

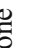

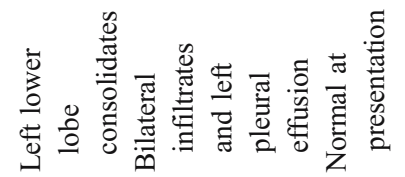

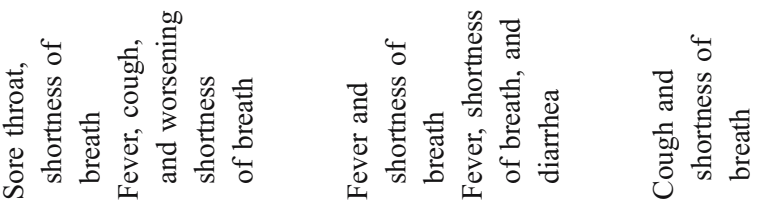

尊

宅言言

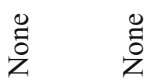

zั zั

气ั

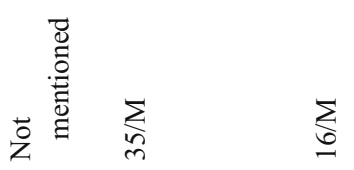

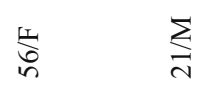

$\sum_{\infty} \quad \overrightarrow{\stackrel{\Xi}{\tilde{\Xi}}}$<smiles>C1CCCCC1</smiles>

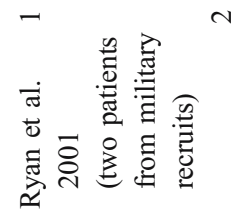

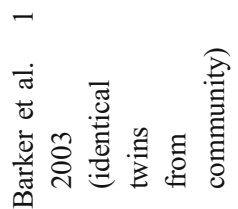

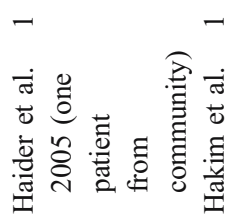

于

$\sqrt{5}$

5

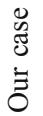


$[11,12]$. However, the relationship between virulence and genotype needs to be explored.

To conclude, the clinical scenario of severe adenovirus pneumonia resembles severe bacterial pneumonia and the two may be indistinguishable on clinical and radiological grounds. Diagnosis of adenovirus infection should be always considered in patients with severe pneumonia with negative cultures and failure to respond to antibiotics. Since this can rapidly progress to respiratory failure, the diagnosis should be looked for early in the course to avoid unnecessary drugs and to apply aggressive supportive measures in the ICU setting which might improve outcome in such patients. More work needs to be done to identify viral determinants and genetic factors triggering severe adenovirus infection in immunocompetent individuals and to better understand the pathophysiology and immunology of such infections. We also need to identify subsets of patients with severe adenovirus who could benefit from antiviral drugs.

\section{References}

1. Carrigan DR (1997) Adenovirus infections in immunocompromised patients. Am J Med 102:71-74

2. Van Der Veen J, Dijkman JH (1962) Association of type 21 adenovirus with acute respiratory illness in military recruits. Am J Hyg 76:149-159
3. Dudding BA, Wagner SC, Zeller JA, Gmelich JT, French GR, Top FH Jr (1972) Fatal pneumonia associated with adenovirus type 7 in three military trainees. N Engl J Med 286:1289-1292

4. From the Centers for Disease Control and Prevention (2001) Two fatal cases of adenovirus-related illness in previously healthy young adults-Illinois, 2000. JAMA 286:782-783

5. Barker JH, Luby JP, Dalley AS, Bartek WM, Burns DK, Erdman DD (2003) Fatal type 3 adenoviral pneumonia in immunocompetent adult identical twins. Clin Infect Dis 37:142-146

6. Haider K, Qadir A, Mujibur R, Judith B, Victoria B (2006) Severe adenovirus infection in an immunocompetent adult-a case report. New York Medical Journal April (sponsored by St. Barnabas Hospital, Bronx, NY)

7. Pingleton SK, Pingleton WW, Hill RH, Dixon A, Sobonya RE, Gertzer J (1978) Type 3 adenoviral pneumonia occurring in a respiratory intensive care unit. Chest 73:554-555

8. Klinger JR, Sanchez MP, Curtin LA, Durkin M, Matyas B (1998) Multiple cases of life-threatening adenovirus pneumonia in a mental health care center. Am J Respir Crit Care Med 157:645649

9. Noppen M, Vanmaele L, Schandevyl W (1986) Severe adenovirus pneumonia in an adult civilian. Eur $\mathrm{J}$ Respir Dis 69:188-191

10. Ribaud P, Scieux C, Fraymuth F, Morinet F, Gluckman E (1999) Successful treatment of adenovirus disease with intravenous cidofir in an unrelated stem cell transplant recipient. Clin Infect Dis 28:690-691

11. Hayder H, Mullbacher A (1996) molecular basis of immune evasion strategies by adenovirus. Immunol Cell Biol 70:67336740

12. Smith CA, Woodruff LS, Kitchingman GR, Rooney CM (1996) Adenovirus-pulsed dendritic cells stimulate human virus-specific T-cell responses in vitro. J Virol 70:6733-6740 